Journal of Computer Science 2 (4): 363-369, 2006

ISSN 1549-3636

(C) 2006 Science Publications

\title{
A Framework for Design of Intelligent Simulation Environment
}

\author{
${ }^{1}$ Ali Azadeh, and ${ }^{2}$ Farid Ghaderi, \\ ${ }^{1}$ Department of Industrial Engineering, Research Institute of Energy Management and Planning \\ ${ }^{2}$ Department of Engineering Optimization, Faculty of Engineering, University of Tehran, Iran
}

\begin{abstract}
This study describes a framework for design and development of intelligent simulation environment. The intelligent simulation environment prescribes an integrated rule-based approach to simulate and optimize a manufacturing system. An intelligent simulation model is a computer program capable of improving its performance by referring to production constraints, system's limitations and desired targets. It is a goal oriented, flexible and integrated approach and produces the optimum solution by referring to an integrated database. The properties and modules of the prescribed intelligent simulation environment are: 1) parametric modeling, 2) flexibility module, 3) integrated modeling, 4) rule-based module, 5) integrated database and 6) learning module. The design phase of the intelligent simulation environment is discussed for a large multi product assembly shop and a heavy continuous rolling mill system.
\end{abstract}

Key words: Intelligent simulation, integrated modeling, rule-based, flexibility module, learning module

\section{INTRODUCTION}

Computer simulations are exquisite tools for modeling and analyzing the true performance of the evolving production systems. An intelligent computer simulation environment would allow the designers of manufacturing systems to predict and provide the means to control the relevant disturbances to an acceptable degree of completeness. It would automatically enable us to foresee the behavior of such systems in normal and increased production situations ${ }^{[1-3]}$. Furthermore, It should intelligently guide us to a smoother and more efficient performance for production systems.

Khoshnevis, Lucker and Adelsberger and Shannon ${ }^{[4-7]}$ discuss the importance of intelligent simulation modeling. Rao et al. and Oren and Zeigler to overcome the inflexible and limited issues of traditional simulation techniques ${ }^{[8-10]}$ propose intelligent knowledge-based modeling. An intelligent simulation environment is a large knowledge integration system, which consist of several symbolic reasoning systems (LISP, PROLOG, etc.) and numerical simulation software. In fact, these studies suggest a framework for integration of numerical simulation, expert system and artificial intelligence techniques.

An integrated computer simulation environment for optimization of production systems is proposed by Batocchio and Franco ${ }^{[11]}$. The prescribed environment is built by referring to the concepts of Theory of Constraints. Khoshnevis and Parisay ${ }^{[12]}$ propose an intelligent simulation environment based on the idea of machine learning and improvement mechanism. They describe the potential of learning in simulation as means for extracting rules to be used in expert systems. Prakash and Shannon ${ }^{[13,14]}$ propose a goal-oriented simulation environment for optimization of manufacturing systems. In a goal-oriented environment, once the system is described and the goals specified, the simulation system drives itself to goal achievement.

Several studies show the applications of intelligent simulation in the area of production systems. These studies discuss the intelligent simulation environment of various control strategies. An integrated knowledgebased model is developed for complex man-machine systems by Azadeh ${ }^{[15]}$. Intelligent simulation environments are also proposed for flexible manufacturing systems, information systems, process plants, just-in-time and aerospace manufacturing systems $^{[16-23]}$.

In this study, an intelligent modeling environment is a flexible, integrated, and knowledge-based framework capable of learning and correcting itself. It is goal oriented and searches for the best solutions by referring to desired target. The importance of an intelligent approach is more evident by noting the properties and requirements of the factory of the future. The challenge is to develop the technology required to achieve a new generation of manufacturing systems. They are not only flexible and computer integrated, but are capable of adapting themselves to necessary changes ${ }^{[24,25]}$. Furthermore, the next production frontier is operating factories as learning laboratories ${ }^{[26,27]}$. An intelligent Simulation environment could aid us overcome the evolving requirements of the factory of the future.

It is therefore the objective of this study to describe an intelligent knowledge-base simulation environment for optimization of the performance of manufacturing systems. An intelligent simulation environment is proposed by integration of: 1) an integrated data base

Corresponding Author: Ali Azadeh, Department of Industrial Engineering, Research Institute of Energy Management and Planning, University of Tehran, Iran 
and modeling, 2) rule-based (goal-oriented) behavior and 3) parametric and flexible structures discussed in this study. The prescribed framework is discussed for a large multi product assembly shop and a heavy continuous rolling mill. Furthermore, only the development process of the intelligent simulation environment and its design elements are described for the two systems and complete modeling process is left for a future extended study. Therefore, the traditional simulation models are developed for the above systems and only the process of translating the traditional models into the prescribed intelligent simulation approach of this study is explained.

Integrated simulation modeling: Integrated modeling environment is an essential aspect of intelligent simulation and is composed of two features: 1) integrated database and 2) integrated modeling. The former requires integrated rather than stationary approach of modeling. Furthermore, all system requirements, limitations, constraints, inputs, interrelationships (with other systems) and feedback are taken into account and modeled. Integrated database is complementary to integrated modeling and is concerned with comprehensive and robust data collection analysis in the system being studied. Moreover, it should be capable of revealing system status based on maintenance and operation activities. Data analysis is an important aspect of a simulation study. There are three types of input data available: 1) The data related to general engineering knowledge of design and manufacturing of the operations and processes, 2) the data from experiences of the specific system being studied and 3) the data collected from a comprehensive time study. These three types of data may be used to assess the hardware failure rates, expected operation times, inspection and maintenance data, etc. The objective of data collection and analysis of an intelligent simulation project must be to integrate these three types of information to get to the best solutions. An integrated database is a key element in developing an intelligent knowledge-based simulation environment.

The components' failures and operation and service times of complex production systems may not be easily predicted due to their stochastic and ambiguous nature. There are two major problems: first, the engineers prefer to deal with absolutes than probabilities and second, they do not collect enough data to make statistical estimates of important parameters such as process times, failure times, etc. Therefore, the data analysis techniques show biased results that may or may not foresee the true nature of such systems. This means the simulation results may not reflect the actual performance of the system being studied.

Therefore, there is a need to understand the stochastic nature of such systems and collect necessary and sufficient data to assess their non-transient point estimates. The necessary and sufficient data means collecting enough data and assessing steady state of point estimates. Also, proper statistical techniques such as Goodness for Fit techniques must be used ${ }^{[28]}$. Therefore, an integrated database in context of an intelligent simulation environment must address the above issues. It must contain robust and meaningful sets of data according to the laws of probability and statistics. It acts as a knowledge base for the simulation environment. Simulation environment retrieves data from integrated database and vice versa. The integrated database must be designed such that any information regarding the system status could be retrieved from it.

Intelligent simulation: It must be noted that each system is atypical and the problem solving approach of each system must be based on Systems Uniqueness Philosophy. This means each intelligent simulation model is unique and requires an approach that dwells on its own contextual requirements. There is a need to adopt a more holistic approach to simulation of manufacturing systems. Moreover, the whole picture must be considered and the trap of dealing with specialties with which we feel comfortable must be avoided. As mentioned, an intelligent simulation environment requires integrated rather than stationary approach of modeling. Consequently, hidden points and bottlenecks are reduced.

Once the objective of the study is specified, the system under study needs to be described. Conceptual models are used to address the states, conditions and operations of the system being studied. Also, system's tolerances, capacity requirements and its interactions with other systems must be modeled. An intelligent simulation model is a set of versatile, flexible and parametric computer programs (Fig. 1). It is composed of an integrated database that by learning process (rulebased module) leads to the best answer or a set of optimum solutions. The rule-based module guides the model toward optimum solutions. The flexibility module defines system's tolerances, capacity requirements and limitations. The intelligent simulation environment of this study is composed of the following features:

It has a flexible structure such that various alternatives and strategies could be retrieved from it. It is composed of parametric rather than numeric simulation programs.

It is a goal-oriented simulation model. It searches for the best solution or a set of optimum answers by referring to actual system's limitations and production constraints. It retrieves data from the integrated database to find the best solution. Also, it inputs the new information to the database for future use. The goal-oriented approach is modeled in a rule-based fashion.

An intelligent simulation model is composed of a learning module. It learns from its previous experiences and creates new rules. The new rules are applied to 
solve new problem or find better solutions. This process is repeated until a best answer is reported. Furthermore, if the new rule or strategy is matched with system's expectations (objectives), it is then chosen as the best answer. The new strategies are stored in the integrated database for the future use.

It is an integrated rather than an isolated approach of simulation modeling. It contains the most important interrelationships, properties and functions of the system being studied. This feature involves globally rather than stationary (local) methods of synthesis. This is quite important in locating hidden points and deficiencies that may exist in production systems. It considers all endogenous and exogenous factors that affect the system under study.

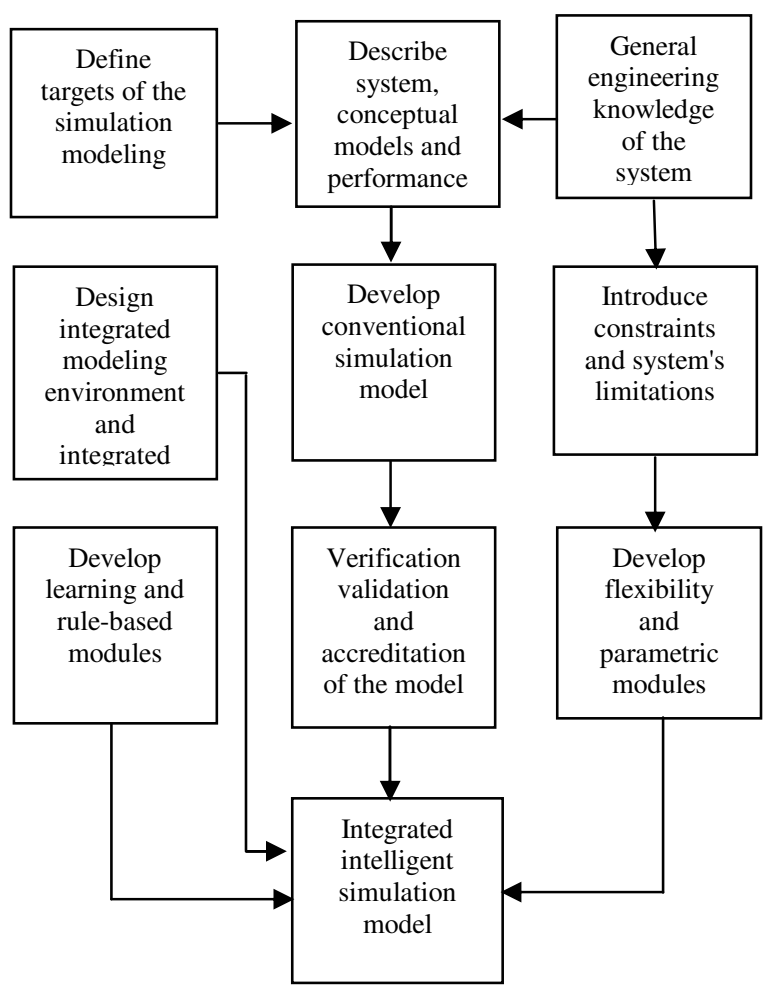

Fig. 1: Design elements of the intelligent simulation environment

First prototype: To show how an intelligent environment could be designed and developed, a large manufacturer of electricmotors was considered. The system being studied is composed of three units: 1) coil operation shop, 2) core insulation shop and 3) final assembly shop. The semi-finished products from coil operation and core insulation shops are required to go under special treatments and are therefore sent to other shops outside the system and are finally routed back to core insulation and final assembly shops, respectively (Fig. 2). There are 21 manual, semi-manual and fully automated workstations. The target was defined as to determine the strategies required meeting the increased demand by 30 percent. Historical data, general engineering knowledge, specifications, layouts were collected systematically. In addition, an identification form was developed for each workstation. It contains valuable information such as process times, buffer size, number of operators and machines. It also reveals machine speed rates and description of operations of a workstation. Performance measures were defined as daily production rates in each the three shops.

Process times were collected by a time study methodology and analyzed through a series of robust statistical techniques. Other information such as machine's speed rates, buffer sizes, etc. were provided by historical data and general engineering knowledge of supervisors. Due to stochastic nature of the processes, the distribution functions of several activities were evaluated. Goodness of Fit techniques was applied to evaluate the best distribution functions for all activities and processes of the assembly shop. An integrated database was designed to contain all of the above information.

The conventional simulation model was developed by AWESIM simulation language ${ }^{[29,30]}$. It contains detailed information about the processes and operations of the assembly shop. To translate the traditional simulation model into the prescribed approach, the interacting systems namely: Insulating Varnish and Machine shops should be modeled into the simulation environment. To create a flexible and parametric environment, a series of Visual SLAM variables and network functions must be modeled into the simulation program. Furthermore, all variables and constants must be changed to global variables and mathematical expressions and constraints should model system's limitations such as production capacity, machine tolerances, etc. The simulation must be capable of generating various alternatives (by changing resources and tolerances) until the optimum solution is reported. The database should be capable of interacting with the simulation environment through a series of READ and WRITE statements. By designing a sequence of rulebased conditions, the model searches for the optimum solution in relation to the defined objective. The learned strategies are stored in the integrated database for the future use and comparative analysis. This process is repeated until the strategies required to increase the throughput by 30 percent are reported. The simulation model must be verified and validated prior to translation into intelligent environment. Therefore, it was verified and validated against the actual assembly shop. Daily production rate was chosen as the validation parameter. The assembly shop was simulated for one working day and repeated 100 times. The outputs of 100 simulation runs were compared with the production rates of 100 working days and hence the null hypothesis $\mathrm{H}_{\mathrm{o}}: \mu_{1}=\mu_{2}$ was tested at $\alpha=0.05$. The results are shown in Table 1. Also, from the independent t-test, we conclude that the average production rates for assembly shop and simulation are equal (at $\alpha=0.05$ ). 


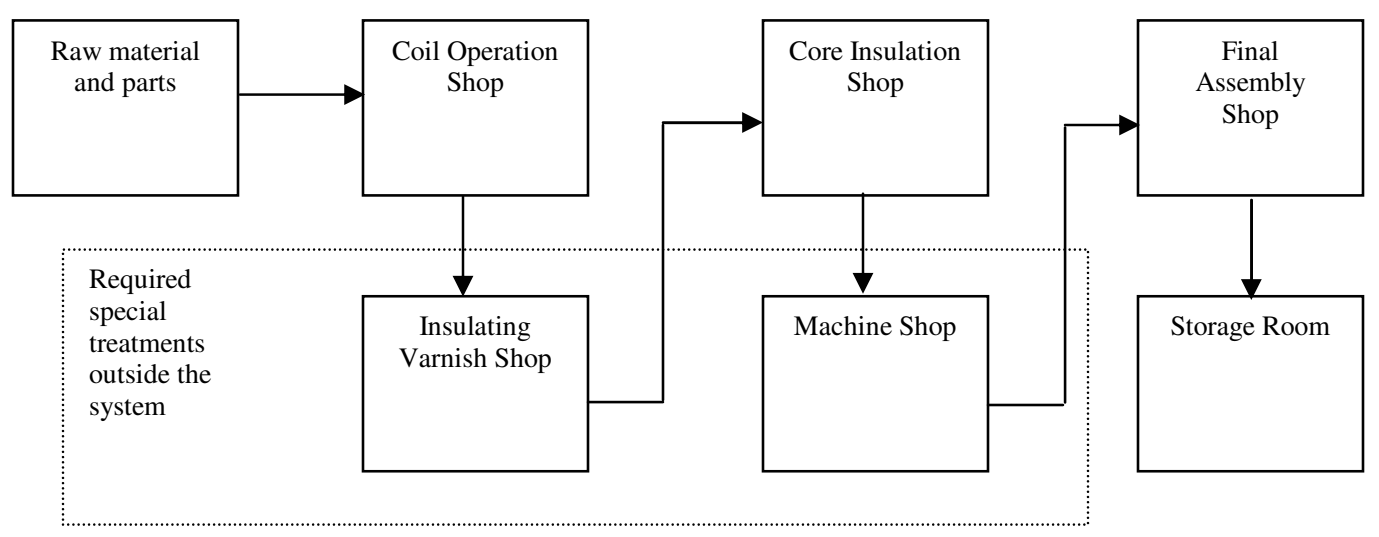

Fig. 2: A general overview of the assembly shop

Table 1: The throughput results for simulation and actual system

\begin{tabular}{lllll}
\hline & No. of observations & Average production & Standard deviation & Confidence limits \\
\hline Assembly & 100 days & 50.97 & 42.87 & {$[42.86,59.37]$} \\
Simulation & 100 days & 46.25 & 40.36 & {$[38.33,54.16]$} \\
\hline
\end{tabular}

Note that the equality of variances of $\mathrm{H}_{\mathrm{o}}: \sigma_{1}{ }^{2}=\sigma_{2}{ }^{2}$ by F-test was tested prior to the t-test. Furthermore, the null hypothesis was accepted at $\alpha=0.05$.

Table 2 shows the classification of various alternatives in unit three of the assembly shop. The number in the boxes below workstation number indicates the number of additional operators to a particular workstation. For example, if one operator is added to workstation number eleven, 17.4 percent increase in production throughput would be expected. The alternatives shown in Table 2 are chosen from a set of more than 25 scenarios. In conventional simulation approach, each scenario is separately designed, modeled, run and evaluated by the user. This approach is a very time-consuming effort and may lead to human errors. In addition, due to complexity of manufacturing systems, a near optimum solution may be introduced. However, by utilization of the intelligent simulation framework (discussed in this study) the user directly obtains the best solution (alternative number 4) without confusion in a shorter time. Furthermore, system limitation is defined as allowance of addition of up to two operators to workstations number $11,12,13$ and 14. Alternative number 4 may be achieved through a series of learned rules and strategies and flexible and integrated structures of the simulation model of the system being studied. Moreover, the intelligent simulation model considers system's limitation and by a rule-base learning mechanism locates the optimum rather than near optimum solution. The reader should note that the percent improvement in throughput remains almost the same after one operator is added to workstations number 11, 12 and 13. However in conventional simulation approach alternative number 5 or 6 may be selected if further analysis is not performed. It means alternatives with the same outcome but higher costs are proposed.
Table 2: Selected alternatives based on conventional simulation results

\begin{tabular}{lllllc}
\hline Alternative & \multicolumn{3}{c}{ Storkstation Number } & \multicolumn{2}{c}{ Percent improvement } \\
Number & 11 & 12 & 13 & 15 & throughput \\
\hline 1 & - & - & - & - & 0.0 \\
2 & 1 & - & - & - & 17.04 \\
3 & 1 & 1 & - & - & 19.6 \\
4 & 1 & 1 & 1 & - & 35.2 \\
5 & 1 & 1 & 1 & 1 & 37.5 \\
6 & 1 & 1 & 1 & 2 & 37.1 \\
7 & 1 & 1 & 2 & 1 & 36.2 \\
8 & 1 & 2 & 1 & 1 & 35.6 \\
9 & 2 & 1 & 1 & 1 & 36.9 \\
\hline
\end{tabular}

Second prototype: The system being studied is a continuous rolling mill line of a large-scale steelmaking factory. Although the workshop is capable of producing various types of profiles, but the major products are profiles 14 and 16. Furthermore, the input of this workshop is steel-bars and the output products are different types of profiles. The steel-bars are transferred from the casting workshop to the storage facility in the Rolling mill workshop. Then, they are charged to a furnace with the production rate of 200 tons per hour. There is a 400-tons cutter after the furnace station. The cutter is used to divide a bar into smaller parts, when the production line is being tested. After the cutter, there are rough mills that are used to shape bars. There are 9 rough mills and each of them is made from two rolls. The mills number $1,3,5,7$ and 9 are positioned horizontally, and the dies number 2, 4, 6 and 8 can be positioned vertically or horizontally according to the type of profile. The shape of a heated steel-bar is changed, when it passes through these mills. After rough mills, there is a 130-tons cutter. In a normal condition it cuts off the head of the bar and in an emergency situation, it divides the bar into the small parts. There are 7 final mills in the next station. Passing through this station, steel-bars take their final shape. 


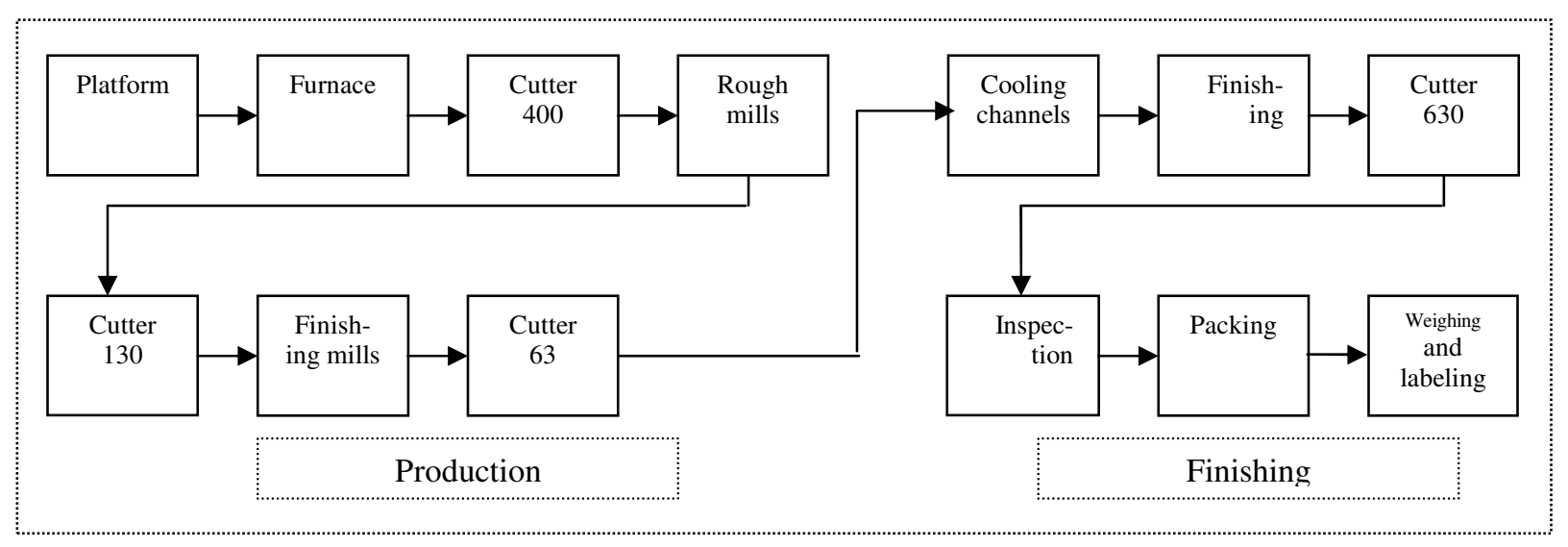

Fig. 3: Overview of the heavy rolling mill system

Table 3: Two-sample analysis results of the rolling mill and simulation

\begin{tabular}{lllll}
\hline & Number of observation & Average throughput rates (tons) & Standard deviation & 95\% Confidence interval \\
\hline Rolling Mill & 11 & 4348.00 & 278.17 & $4,158.28,4,537.72$ \\
Simulation & 12 & 4346.67 & 211.25 & $4,210.80,4,482.54$ \\
\hline
\end{tabular}

Table 4: The sensitivity of furnace's capacity vs. daily production rate of the line

\begin{tabular}{lllllll}
\hline Furnace's capacity (tons/hour) & 150 & 180 & 200 & 220 & 250 & More than 250 \\
Monthly production rate (tons) & $84,593.9$ & $112,936.9$ & $116,261.8$ & $120,603.2$ & $123,100.4$ & About 123,200.0 \\
\hline
\end{tabular}

There is a 63-tons cutter in the next station that divides the bars into three parts. Then the bars are transferred to a cooling channel and the finishing station. There are two parallel machines in the finishing station to increase the line efficiency. Bars are transferred to a 630-tone cold cutter and are divided to 12-meter parts. Then, they are moved to the inspection section. Afterwards, they are weighted, labeled and moved to the storage. The parts that pass the inspection are moved to the packing station composed of two parallel machines. The existence of two parallel finishing and packing machines has created two left and right lines from the beginning of the finishing station. Figure 3 shows the overview of the rolling mill system.

AWESIM Simulation language was used to build the traditional model. The target was defined as to optimize the line without any additional resources. The simulation model of the rolling mill workshop is very similar to a Just in Time (JIT) production model. If the rolling mill workshop is divided into storage, furnace, finishing, cutting and inspection departments, the operation of each part will depend on the last and the next parts. Therefore, the simulation model for this system is built according to the JIT logic of the actual system and considers the available capacity between stations instead of the economic batch. Two different criteria are chosen to examine the validation of the simulation model: 1) the throughput of the furnace, and 2 ) the production rate of the Rolling mill line. The simulation was run for 5 days and repeated 12 times. Measured values were examined by the $t$-test for the two systems. The test has proved the statistical similarity between the model and the system throughputs. Furthermore, from the t-test it is concluded that the average production rates for the rolling mill and simulation systems are statistically equal (at $\alpha=0.05$ ). As an example, the comparison of the throughput of the furnace for the two systems is shown in Table 3. The equality of variance was tested prior to the t-test.

The simulation model was run for a period of six months. The conditions of workstations are monitored and since the furnace is the most important station in this workshop, its condition was analyzed in through various simulation runs. The results showed that the furnace cannot feed the line properly, and to reach a balanced production line the capacity of this station should be increased to an optimum level. Therefore, the bottleneck in the line is the furnace station. To handle this issue, we need to know which station would be the next bottleneck? Also, if the furnace problem were resolved, by how much the production rate would increase? Finally, what is the optimum capacity of the furnace? This is exactly how the rule base module works in an intelligent simulation environment. It would automatically generate the solutions to the above questions through a series of if $>$ then mechanisms.

To find answers for the above questions, the conventional simulation model was ran several times and the results were analyzed. In the first attempt, the furnace capacity is supposed to be unlimited. In this case it was expected that the line would become fully utilized. But the simulation results showed 17.9 percent of idle time for the furnace and the production line. This idle time was caused by the failure of another station in 
Table 5: The properties of the integrated simulation environment

\begin{tabular}{|c|c|c|}
\hline Property/Module & Definition & Example in Visual SLAM \\
\hline Parametric simulation & $\begin{array}{l}\text { Modeling of variable and constants in terms of global } \\
\text { variables }\end{array}$ & $\begin{array}{l}X X[1] \\
\text { ARRAY[1,4] }\end{array}$ \\
\hline Flexibility module & $\begin{array}{l}\text { Modeling of system and sub-system limitations in } \\
\text { terms of mathematical expressions and constraints }\end{array}$ & $\begin{array}{l}\text { Daily production rate: } \operatorname{LL}[1]<=100 \\
\text { Machine speed rate: } 4<=\mathrm{LL}[2]<=6 \\
\text { Service time: } \operatorname{RNORM}(11,2)\end{array}$ \\
\hline Integrated modeling & $\begin{array}{l}\text { Modeling of all activities (operation, maintenance, } \\
\text { inspection), interacting systems and exogenous } \\
\text { factors }\end{array}$ & $\begin{array}{l}\text { In the examples shown in this study, operation, } \\
\text { maintenance, inspection activities and } \\
\text { interacting systems should be modeled }\end{array}$ \\
\hline Rule-based module & $\begin{array}{l}\text { Whether a sets of conditions is satisfied in terms of if } \\
>\text { then }\end{array}$ & $\begin{array}{l}\text { ACTIVITY, } 1,0, \mathrm{LL}[1]<=\mathrm{LL}[4], \mathrm{A} 1 \\
\text { ACTIVITY,2,0, , A2 }\end{array}$ \\
\hline Integrated database & $\begin{array}{l}\text { A database containing information about service, } \\
\text { arrival and machine rates, process times, buffer } \\
\text { capacity, etc. Also, new learned data is stored here }\end{array}$ & $\begin{array}{l}\text { A database designed in EXCEL to interact } \\
\text { with the simulation through a series of WRITE } \\
\text { and READ statements }\end{array}$ \\
\hline Learning module & $\begin{array}{l}\text { It is a process of learning and storing information in } \\
\text { the integrated database by combination and } \\
\text { utilization of the above properties and modules }\end{array}$ & $\begin{array}{l}\text { IF XX[4] }=150 \text { THEN XX[5] }=84593.9 \\
\text { IF XX[4] }=180 \text { THEN XX[5] }=112936.9 \\
\text { NNQ(I)<NNQ(J) THEN SELECT J }\end{array}$ \\
\hline
\end{tabular}

the line. Another run showed that the packing station would be the next bottleneck, if the furnace capacity increases. It was also predicted that if the furnace capacity increases, the production rate of the line would increase by about 46 percent.

In order to determine the optimum capacity of the furnace, the simulation model was run with different capacities and the results were evaluated. This analysis showed that the increase in the furnace capacity up to 250 tons per hour would have a positive effect on the production rate. But after 250 tons per hour, the production rate remains almost constant. Therefore, increasing the furnace capacity to more than 250 tons per hour does not have logical and economic justification. Table 4 illustrates the line's production rate with different furnace capacities. In fact, with an ideal furnace (250 tons/hour), the monthly production rate increases to about 123,000 tons or 52 percent increase in the throughput of the shop would be observed.

The readers should note that the traditional simulation approach required several individual runs and analysis to assess the major bottleneck (furnace) in the line, some of which were reported in the last paragraph. This challenge was also repeated to obtain the results of Table 4 , which is a very time consuming and complex process. However, the development and modeling of an intelligent simulation environment for the rolling mill would automatically generate the results of Table 4 by integration of the prescribed modules.

In order to integrate the model and increase it's flexibility, it is made of 13 different networks, and each network represents an aspect of the production process. Maintenance networks should be designed to define the downtimes caused by failures and maintenance. The model is developed such that it could be integrated with other workshops of the factory. Therefore, the integrated modeling property of the prescribed environment is easily achieved for the line. All variable and constants related to operation, maintenance and inspection activities must be translated into AWESIM global variables (parametric module).
A set of mathematical constraints and expressions need to be developed to express platform and furnace capacity and furnace speed tolerance to represent the flexibility modules. The integrated database would only act as knowledge base for the learned strategies. Furthermore, as new alternatives are chosen and tested and checked against each other by the intelligent model, the new values are stored in the database for the future use. For example, if the intelligent simulation (through various intelligent and automatic runs) identifies furnace as the major bottleneck, the learning module tries to improve its performance by changing its capacity. The new learned strategies are then stored in the database. This is achieved by trials and errors and by using READ and WRITE statements of Visual SLAM.

\section{CONCLUSION}

This study presented a conceptual framework for development of intelligent simulation in manufacturing systems. The prescribed framework was modeled and discussed for two actual manufacturing systems. An intelligent simulation model must be flexible so that various alternatives and strategies could be retrieved from it. In addition, it must incorporate management and economics factors to drive to the best solutions ${ }^{[21]}$.

Several factors must be noted to accomplish an intelligent simulation model. First, to implement its findings, the operations, processes and activities of the system being studied must be studied. Second, a more holistic simulation approach to problems of production systems must be incorporated. The distinct feature of the prescribed approach is integration of flexible structure, rule-based module, integrated database and modeling and parametric simulation modeling (Table 5). The advantage of this approach is as follows:

* It searches for the best solution or a set of optimum answers by a rule-based mechanism.

* An intelligent simulation model considers actual system's limitations and constraints. 
* The system being studied could be simulated without much confusion in a shorter time.

* The results of an intelligent simulation model are practical and may be implemented in the system being studied.

* It is an integrated approach that considers all endogenous and exogenous factors affecting system being studied.

\section{REFERENCES}

1. Azadeh, M.A. and M. Nejati, 1999. Analyzing a press shop via computer simulation. Annu. Conf. on Industrial Engineering, Sharif University of Technology, Tehran, Iran.

2. Azadeh, M.A., 1999. An algorithm for accomplishment of simulation projects in developing countries: The case of an assembly shop. Proc. of the Fourth Intl. Conf. on Reliability, Maintainability and Safety (ICRMS'99), Shanghai, China.

3. Azadeh, M.A., 1988. Simulation of consolidated performance of man-machine systems. Proc. of Europ. Ergonomics Society and Belgium Society of Mechanical Engineering, Brussels, Belgium.

4. Khoshnevis, B., 1994. Discrete Systems Simulation. New York, McGraw Hill.

5. Lucker, P.A. and H.H. Adelsberger, 1986. Intelligent simulation environment. Simulation Series, 17: 1. The Society for Computer Simulation.

6. Shannon, R.E., 1988. Knowledge-based simulation techniques for manufacturing. Intl. J. Production Research, 26: 953-973.

7. Shannon, R.E., 1986. Intelligent simulation environment. Proc. of Intelligent Simulation Environment, pp: 150-156, San Diego, CA.

8. Rao, M., T.S. Jiang and J.P. Tsai, 1990. Integrated intelligent simulation environment. Simulation, 54: 291-295.

9. Rao, M., T.S. Jiang, J.P. Tsai and T.M. Jiang, 1989. Intelligent computing environment for scientific and engineering applications. Mathematics and Computers in Simulation, 31: 6.

10. Oren, T.I. and B.P. Zeigler, 1988. Artificial intelligence in modeling and simulation: Direction to Explore. Simulation, 50: 131-134.

11. Batocchio, A. and G.N. Franco, 1998. The theory of constraints integrate to computer simulation to analyze the viability of production systems. Proc. of First Intl. Symp. on Climbing and Walking Robot, Brussels, Belgium.

12. Khoshnevis, B. and S. Parisay, 1993. Machine learning and simulation-application in queuing systems. Simulation, 61: 294-302.

13. Prakash, S., R.E. Shannon and S. Sheppard, 1989. Goal directed simulation environment: A prototype. Proc. of Summer Computer Simulation Conf., pp: 545-549.

14. Prakash, S. and R.E. Shannon, 1993. Development of a goal directed simulation environment for part manufacturing systems. Simulation, 61: 103-115.
15. Azadeh, M.A., 1993. Modeling and simulations of integrated information processing and operators' decision styles to enhance the reliability of nuclear power plants. Ph.D. Thesis, University of Southern California, Los Angeles, USA.

16. Benjaafar, S., 1992. Intelligent simulation for flexible manufacturing systems: An integrated approach. Computers and Industrial Engineering, 22: 297-311.

17. Sakthivel and Agarwal, 1992. Knowledge-based model construction for simulating information systems. Simulation, 59: 223-236.

18. Sztrimbely, W.M. and P.J. Weymouth, 1991. Dynamic process plant simulation and scheduling: An expert system approach. Simulation, 56: 175178.

19. Manivannan, S. and C.D. Pegden, 1990. A rulebased simulator for modeling just-in-time manufacturing systems. Simulation, 55: 109-117.

20. Pritsker, A.A.B., 1985. Decision support systems for engineers and scientists. Intl. Computer Graphics User Show, London England.

21. Azadeh, M.A., 2001. Design of an intelligent simulation environment for manufacturing systems. Proc. of the Third Intl. ICSC Cong. on World Manufacturing Cong., Symp. on Manufacturing Systems (ISMS'2001), 24-27 Sep., at the Center for Integrated Manufacturing Studies, Rochester, New York.

22. Azadeh, M.A., 1999. Integrated methods for improvement of complex manufacturing systems in twenty first century. Proc. of the 1 st Conf. on Fundamental and Industrial Research Achievements, Nov. 29 to Dec. 6 Faculty of Engineering, University of Tehran, Iran.

23. Azadeh, M.A., 1999. An intelligent computer simulation framework for modeling assembly shops. Proc. of the 26th Intl. Conf. on Computers and Industrial Engineering, Melbourne, Australia.

24. Solberg, J.J., 1987. The next step: Intelligent manufacturing systems. Technical Report, Purdue University.

25. Upton, D.M., 1995. What really makes factories flexible? Harvard Business Rev., Jul.-Aug., pp: 7484.

26. Leonard-Barton, D., 1992. The factory as a learning laboratory. Sloan Management Review/Fall, pp: 23-38.

27. Jackson, R.H.F. and A.W.T. Jones, 1987. An architecture for decision making in the factory of the future. Interfaces, 17: 15-28.

28. Law, A.M. and W.D. Kelton, 1982. Simulation Modeling and Analysis. New York, McGraw Hill.

29. Pritsker, A.A.B., J.J. O'Reilly and D.K. LaVal, 1997. Simulation with Visual SLAM and AWESIM. New York, John Wiley.

30. Azadeh, M.A., 2000. Optimization of a heavy continuous rolling mill system via simulation. Proc. of the 7th Intl. Conf. on Industrial Engineering and Engineering Management (IE\&EM'2000), Nov. 28-30, Guangzhou, China. 